\title{
THE SWEDISH BED-STATE
}

\author{
BY \\ GUNNAR DAHLBERG \\ (Statens' Rasbiologiska Institut, Uppsala)
}

In view of the paucity of reliable civilian statistics with respect to hospital accommodation requirements, my friend Professor Lancelot Hogben, F.R.S. has suggested that a brief statement of the most recent data separately available for Sweden as a whole and for Stockholm might prove serviceable to Regional Authorities responsible for the implementation of the new National Health Service in Britain. Those exhibited in the ensuing tables refer to all classes of institutional treatment other than psychiatric cases. They therefore exclude patients in mental hospitals and in establishments for the mental defective. They also exclude inmates of institutions for inebriates.

Table I, which cites beds available in all classes of institutions with the reservation stated above, refers to Sweden as a whole for the year 1944, at the end of which the total population of the kingdom was $6,597,348$, including in all 860,268 married women of child-bearing (under 45 years) age and 1,398,388 children under 15 years of age. On the last day of the year, the total number of beds available
TABLE I

SWEDISH BeD-STATE, 1944

\begin{tabular}{|c|c|c|}
\hline Institutions & $\begin{array}{l}\text { No. of beds } \\
\text { equipped on } \\
\text { the last day } \\
\text { of the year }\end{array}$ & $\begin{array}{c}\text { Mean daily } \\
\text { no. occupied } \\
\text { throughout } \\
\text { the year }\end{array}$ \\
\hline $\begin{array}{l}\text { General hospitals } \\
\text { Cottage hospitals } \\
\text { Wards in charitable }\end{array}$ & $\begin{array}{r}21,073 \\
1,691\end{array}$ & $\begin{array}{r}20,390 \\
1,666\end{array}$ \\
\hline $\begin{array}{l}\text { institutions } \\
\text { Private nursing homes }\end{array}$ & $\begin{array}{l}1,845 \\
1,117\end{array}$ & $\begin{array}{l}1,623 \\
1,026\end{array}$ \\
\hline $\begin{array}{l}\text { sanatoria } \\
\text { Fever hospitals } \\
\text { Tuberculosis institutions } \\
\text { Homes for incurable sick } \\
\text { Miscellaneous other } \\
\text { Lying-in hospitals }\end{array}$ & $\begin{array}{l}2,617 \\
6,746 \\
9,104 \\
5,966 \\
975 \\
2,940\end{array}$ & $\begin{array}{r}1,427 \\
4,659 \\
8,066 \\
5,350 \\
890 \\
3,066\end{array}$ \\
\hline Total & 54,074 & 48,163 \\
\hline $\begin{array}{l}\text { Total excluding maternity } \\
\text { cases } . . . . .\end{array}$ & 51,134 & 45,097 \\
\hline
\end{tabular}

TABLE II

STOCKHOLM BED-STATE, 1944

\begin{tabular}{|c|c|c|c|c|c|c|c|c|}
\hline \multirow{2}{*}{\multicolumn{3}{|c|}{ Beds available for }} & \multicolumn{2}{|c|}{ Municipal institutions } & \multicolumn{2}{|c|}{ Other major establishments } & \multicolumn{2}{|c|}{ Total } \\
\hline & & & $\begin{array}{l}\text { Beds avail- } \\
\text { able on last } \\
\text { day of year }\end{array}$ & $\begin{array}{l}\text { Mean daily } \\
\text { no. of beds } \\
\text { occupied }\end{array}$ & $\begin{array}{l}\text { Beds avail- } \\
\text { able on last } \\
\text { day of year }\end{array}$ & $\begin{array}{l}\text { Mean daily } \\
\text { no. of beds } \\
\text { occupied }\end{array}$ & $\begin{array}{c}\text { Beds } \\
\text { avail- } \\
\text { able }\end{array}$ & $\begin{array}{c}\text { Mean } \\
\text { daily } \\
\text { no. }\end{array}$ \\
\hline $\begin{array}{l}\text { Medical (excluding tho } \\
\text { Surgical (ditto) ... } \\
\text { Tuberculosis } \ldots \\
\text { Children's diseases } \\
\text { Infectious diseases } \\
\text { Ophthalmic . } \\
\text { Ear, nose, and throat } \\
\text { Women's diseases } \\
\text { Venereal diseases } \\
\text { Neurological cases } \\
\text { Chronic sick .. } \\
\text { Orthopaedic . } \\
\text { Radium treatment } \\
\text { Maternity cases .. }\end{array}$ & $\begin{array}{l}\text { e ci } \\
. \\
. \\
. \\
. \\
\therefore \\
\therefore \\
\therefore \\
\therefore \\
\therefore \\
\therefore\end{array}$ & $\begin{array}{l}\text { below) } \\
. . \\
. \\
. \\
. \\
. \\
. \\
. \\
\cdots \\
\therefore \\
. \\
. \\
.\end{array}$ & $\begin{array}{r}1,212 \\
796 \\
884 \\
155 \\
707 \\
40 \\
83 \\
155 \\
320 \\
1,585 \\
76 \\
11 \\
395\end{array}$ & $\begin{array}{r}1,136 \\
723 \\
724 \\
113 \\
484 \\
23 \\
74 \\
157 \\
259 \\
1,365 \\
46 \\
10 \\
363\end{array}$ & $\begin{array}{r}698 \\
686 \\
\overline{231} \\
-82 \\
89 \\
197 \\
87 \\
80 \\
190 \\
171 \\
138 \\
132\end{array}$ & $\begin{array}{r}632 \\
641 \\
207 \\
51 \\
76 \\
205 \\
75 \\
66 \\
187 \\
168 \\
135 \\
140\end{array}$ & $\begin{array}{r}1,910 \\
1,482 \\
884 \\
386 \\
707 \\
122 \\
172 \\
352 \\
407 \\
80 \\
1,775 \\
247 \\
149 \\
527\end{array}$ & $\begin{array}{r}1,768 \\
1,364 \\
724 \\
320 \\
484 \\
74 \\
150 \\
362 \\
334 \\
66 \\
1,552 \\
214 \\
145 \\
503\end{array}$ \\
\hline Total & & . & 6,419 & 5,477 & 2,781 & 2,583 & 9,200 & 8,060 \\
\hline \multicolumn{3}{|c|}{ Total, excluding maternity cases } & 6,024 & 5,114 & 2,649 & 2,443 & 8,673 & 7,557 \\
\hline
\end{tabular}


TABLE III

Mean Monthly Numbers of Beds occupied in MunictPal Hospitals-Stockholm, 1944 (maxima in italics)

\begin{tabular}{|c|c|c|c|c|c|c|c|c|c|c|c|c|c|c|c|c|}
\hline Medical units for & & Jan. & Feb. & Mar. & April & May & June & July & Aug. & Sept. & Oct. & Nov. & Dec. & $\begin{array}{c}\text { Average } \\
1944\end{array}$ & $\begin{array}{c}\text { Beds } \\
\text { avail- } \\
\text { able }\end{array}$ & $\begin{array}{c}\text { Ditto } \\
\text { per } \\
1,000 \\
\text { popn. }\end{array}$ \\
\hline $\begin{array}{l}\text { Internal diseases .. } \\
\text { Neurological cases } \\
\text { Surgical cases } \\
\text { Neurosurgery } \\
\text { Women's diseases } \\
\text { Ophthalmic cases } \\
\text { Ear, nose, and throat } \\
\text { Venereal diseases .. } \\
\text { Radium . . } \\
\text { Children's medical } \\
\text { Children's surgical } \\
\text { Infectious diseases } \\
\text { Orthopaedic cases } \\
\text { Surgical tuberculosis } \\
\text { Phthisis } \\
\text { Chronic sick } \quad . . \\
\text { Maternity cases } \quad . .\end{array}$ & $\begin{array}{l}\ldots \\
\cdots \\
\cdots \\
\cdots \\
\cdots \\
\cdots \\
\cdots \\
\cdots \\
\cdots \\
\cdots \\
\cdots \\
\cdots\end{array}$ & \begin{tabular}{|r|r|}
1,253 \\
13 \\
839 \\
27 \\
149 \\
23 \\
23 \\
72 \\
279 \\
6 \\
213 \\
213 \\
35 \\
423 \\
47 \\
57 \\
642 \\
1,785 \\
291
\end{tabular} & $\begin{array}{r}1,272 \\
15 \\
878 \\
25 \\
158 \\
26 \\
77 \\
289 \\
11 \\
220 \\
34 \\
465 \\
49 \\
57 \\
610 \\
1,800 \\
308\end{array}$ & \begin{tabular}{|r|}
1,298 \\
20 \\
828 \\
30 \\
163 \\
23 \\
76 \\
291 \\
10 \\
203 \\
30 \\
479 \\
49 \\
64 \\
593 \\
1,813 \\
358 \\
\end{tabular} & $\begin{aligned} 1,306 \\
19 \\
803 \\
28 \\
156 \\
24 \\
71 \\
261 \\
11 \\
204 \\
38 \\
510 \\
40 \\
65 \\
590 \\
1,819 \\
332\end{aligned}$ & $\begin{array}{r}1,295 \\
16 \\
792 \\
27 \\
154 \\
23 \\
77 \\
255 \\
10 \\
202 \\
36 \\
473 \\
40 \\
69 \\
627 \\
1,757 \\
348\end{array}$ & $\begin{array}{r}1,158 \\
16 \\
770 \\
24 \\
134 \\
24 \\
75 \\
206 \\
10 \\
178 \\
28 \\
427 \\
31 \\
69 \\
662 \\
1,760 \\
356\end{array}$ & $\begin{array}{r}1,009 \\
13 \\
682 \\
22 \\
127 \\
9 \\
44 \\
44 \\
200 \\
10 \\
146 \\
21 \\
310 \\
33 \\
65 \\
702 \\
1,688 \\
336\end{array}$ & $\begin{array}{r}994 \\
8 \\
700 \\
26 \\
131 \\
15 \\
49 \\
188 \\
11 \\
134 \\
33 \\
295 \\
35 \\
62 \\
702 \\
1,663 \\
329\end{array}$ & $\begin{array}{r}1,134 \\
12 \\
753 \\
30 \\
152 \\
24 \\
69 \\
238 \\
10 \\
194 \\
24 \\
364 \\
47 \\
66 \\
683 \\
1,670 \\
350\end{array}$ & $\begin{array}{r}1,196 \\
19 \\
813 \\
29 \\
160 \\
27 \\
74 \\
273 \\
11 \\
215 \\
31 \\
473 \\
52 \\
67 \\
677 \\
1,681 \\
358\end{array}$ & $\begin{array}{r}1,239 \\
21 \\
844 \\
31 \\
157 \\
25 \\
74 \\
270 \\
11 \\
229 \\
32 \\
454 \\
66 \\
68 \\
694 \\
1,717 \\
356\end{array}$ & $\begin{array}{r}1,196 \\
17 \\
773 \\
26 \\
127 \\
18 \\
68 \\
241 \\
10 \\
214 \\
29 \\
439 \\
63 \\
65 \\
681 \\
1,754 \\
340\end{array}$ & $\begin{array}{r}1,196 \cdot 6 \\
15 \cdot 8 \\
789 \cdot 4 \\
27 \cdot 1 \\
147 \cdot 6 \\
21 \cdot 7 \\
68 \cdot 8 \\
249 \cdot 3 \\
10 \cdot 3 \\
195 \cdot 5 \\
31 \cdot 0 \\
425 \cdot 9 \\
46 \cdot 0 \\
64 \cdot 7 \\
655 \cdot 0 \\
1,741 \cdot 8 \\
339 \cdot 1\end{array}$ & \begin{tabular}{|r|}
1,312 \\
31 \\
959 \\
28 \\
155 \\
40 \\
83 \\
320 \\
11 \\
\\
311 \\
707 \\
76 \\
62 \\
822 \\
1,585 \\
455
\end{tabular} & $\begin{array}{l}2.10 \\
0.05 \\
1.58 \\
0.04 \\
0.31 \\
0.11 \\
0.16 \\
0.49 \\
0.06 \\
0.47 \\
1.08 \\
0.12 \\
0.09 \\
1.26 \\
2.42 \\
0.69\end{array}$ \\
\hline Total & & 6,154 & 6,294 & 6,328 & 6,277 & 6,201 & 5,928 & 5,417 & 5,375 & 5,820 & 6,516 & 6,288 & 6,061 & $6,025 \cdot 6$ & 6,957 & $11 \cdot 03$ \\
\hline
\end{tabular}

per thousand total population was $8 \cdot 2$. The corresponding mean daily figure of beds occupied is $7 \cdot 3$. For further reference it may be well to specify that the figures cited for general hospitals exclude wards for special classes of patients (children, maternity, tuberculosis patients and convalescents) budgeted in special institutions as indicated thereunder.

For Stockholm available figures distinguish more explicitly between number of beds available for and occupied by different types of cases, as exhibited in Table II. The estimated city population on the last day of the year (1944) was 654,864 , of which 95,563 were married women of child-bearing age and 107,222 children under 15 years of age. The figures for beds available (at the end of the year) and mean daily number of beds occupied are respectively 14 per thousand and $12 \cdot 3$ per thousand, if we take the city population as the true population at risk. However, we have to bear in mind that the middle columns, referring to establishments (state and private) other than municipal hospitals, include provision for patients not domiciled in the city.

Against the background of information relating to age and incidence of diseases, breakdown of cases in Table II provides some guidance for activi elsewhere, if vital statistics of the age structure of the populations at risk are to hand. Any estimate of a safety margin between beds equipped and number constantly sick must also take stock of seasonal fluctuations; and it is possible to make such a breakdown of the figures for Stockholm, as shown in Table III. The figures in the latter do not include 330 cases referable to two hospitals which have not furnished statistics, but are otherwise complete. 